\title{
Design High-Barrier and Environmentally Degradable FDCA-Based Copolyesters: Experimental and Theoretical Investigation
}

Han Hu, a Jiayi Li, b Ying Tian, ${ }^{\text {a }}$ Shenggan Luo, b Jinggang Wang, a Wu Bin Ying, a

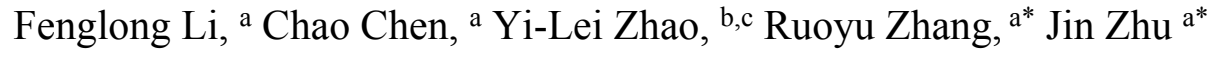

${ }^{a}$ Key Laboratory of Bio-based Polymeric Materials Technology and Application of Zhejiang Province, Ningbo Institute of Materials Technology and Engineering, Chinese Academy of Sciences, Ningbo 315201, People's Republic of China

b State Key Laboratory of Microbial Metabolism, Joint International Research Laboratory of Metabolic \& Developmental Sciences, School of Life Sciences \& Biotechnology, Shanghai Jiao Tong University, Shanghai, 200240, China.

${ }^{c}$ Department of Chemistry and Biochemistry, University of California, Los Angeles, California 90095, United States

Corresponding authors:

zhangruoy@nimte.ac.cn (Ruoyu Zhang);

jzhu@nimte.ac.cn (Jin Zhu)

\section{Contents:}

Total number of pages: 9

Total number of equations: 6

Total number of Figures: 3

Total number of Tables: 7 


\section{Experimental Section}

\section{Quantum Mechanical Calculations}

Conformational search was performed using xTB program developed by the Grimme group. ${ }^{1}$ During the hydrolysis reaction in PBS solution, PNDF50 and PNDF65 was built, in which the ratio of FDCA to aliphatic diacid is set at 1:1 and 2:1, respectively. During the enzymatic degradation, substrate PNDF50 was chosen for simplification. To obtain all stable configurations on the potential energy surface of the PNDF, more than 2000 possible initial geometries of PNDF were generated for conformational search using xtb software version 6.3. ${ }^{1}$ Then these structures were optimized using semi-empirical quantum method at the PM6-DH3 level using MOPAC2016. ${ }^{2}$ These initial geometries in a rough level conformation with relatively low energy and different geometry were screened out by Molclus. ${ }^{3}$ The structure of each species was submitted for precise geometry optimization at M06-2X/6-31+G(d,p) level in gas phase or with SMD implicit solvation model for aqueous solution ${ }^{4}$ using Gaussian 16 software $^{5}$, followed by frequency calculation at the same theoretical level. For conformational search of the substrate, the RMSD threshold was set for $0.5 \AA$, and no constraint was used.

\section{DFT calculations of PNDF hydrolysis}

Geometry optimizations were performed with B3LYP functional ${ }^{6}$ including DFTD3 dispersion correction ${ }^{7}$ and $6-31 \mathrm{G}(\mathrm{d}, \mathrm{p})$ basis set. Vibrational frequency analyses were performed at the same level of theory, to ensure local minima or first-order saddle points, and the free energies were calculated for $298.15 \mathrm{~K}$ and $1.0 \mathrm{~atm}$. In addition, the intrinsic reaction coordinates (IRC) calculations were carried out to identify transition states and immediate reactants and products. ${ }^{8}$ to improve the accuracy of the calculations, single-point energy calculations were performed on the optimized structures using a larger basis set $6-311+G(d, p)$ were performed on the optimized geometries and solvation model based on density (SMD) ${ }^{4}$ solvation correction.

\section{Setup of systems and Molecular dynamics Simulation}

The starting crystal structure of the CALB was obtained from the protein data bank (PDB code: 1TCA). The conformation with the lowest energy was then docked into the CALB using Autodock Vina. ${ }^{9}$ structural adjustments were made to avoid bad steric contacts. DFT optimized structure of each substrate was docked to the binding site. For the docking of the complex conformations, rigid docking was applied (i.e., no bond can rotate). We reported and used the lowest energy docking pose for further studies. The parameters of substrate PBNF were generated with the RESP ${ }^{10}$ method and RHF/6-31* calculation ${ }^{11}$, to generate the bonds, angles, dihedral angles and van der Waals radii 
parameters for the substrate by using the Antechamber package. Following the steps above, three complex systems, PNDF-CALB were set up. Files for complexes were prepared with the tleap module of AMBER $18 .{ }^{12} \mathrm{MD}$ simulations were performed on PNDF-CALB complexes using the AMBER $f f 14 S B$ force field. ${ }^{13}$ The protonation states of the titratable residues (such as His, Glu, and Asp) in the complex were determined at $\mathrm{pH}$ consisting of experimental situation using propka. ${ }^{14}$ Except for Asp134, which was protonated, all acidic residues were negatively charged, while Lys and Arg residues remained positively charged. The only histidine, His 224 of the catalytic triad, was singly protonated at $\mathrm{N}_{\delta}$. Counterions $\left(\mathrm{Na}^{+}\right.$and $\left.\mathrm{Cl}^{-}\right)$were added for the maintenance of system neutrality. The complexes were placed in a truncated octahedral box of water molecules, extending $12.0 \AA$ along each dimension. Long-range electrostatic interactions were calculated with the Particle-Mesh-Ewald (PME) method, ${ }^{15}$ and van der Waals interactions were truncated within $12 \AA$. The time interval was set as 2 fs, and the SHAKE ${ }^{16}$ algorithm was used to constrain the bonds-connecting hydrogen atoms. The entire system was first minimized and heated up to $298 \mathrm{~K}$ before the production process. Structural analyses, such as root-mean-square deviation (RMSD), distance analysis, and clustering analysis, were conducted with the CPPTRAJ tool, implemented in Amber 18.

\section{Non-covalent interaction (NCI) analysis}

In this study, independent gradient model (IGM) ${ }^{17}$ analysis were carried out with the Multiwfn 3.7 program $^{18}$. Molecular plots were visualized by the VMD 1.9.3 program. ${ }^{19}$ The IGM analysis depends on the topological characteristics of the electron density, $\rho$. The IGM descriptor $\delta g^{\text {inter }}$ is given by the difference between the first derivatives of the charge densities for the total system and the fragments (eqs. S1):

$$
\delta \mathrm{g}(\mathrm{r})^{\text {inter }}=\left|\nabla \rho^{\mathrm{IGM}, \text { inter }}\right|-|\nabla \rho|
$$

$\delta \mathrm{g}^{\text {inter }}>0$ indicates the presence of weak interactions, and the magnitude of the descriptor at a point in space indicates the strength of the interaction.

\section{Wavefunction analysis}

The prediction of reactive sites between nucleophile and electrophile was studied by the Fukui function analysis with Hirshfeld charges. ${ }^{20}$ The Fukui function was used to predict reactive sites at the B3LYP-D3/def-TZVP level of theory. Fukui function is defined as eqs. S2 :21,22

$$
f(r)=\left[\frac{\partial \rho(r)}{\partial N}\right]_{v}
$$


where $\mathrm{N}$ is number of electrons in present system, the constant term in the partial derivative is external potential. For a given molecular system, Fukui function was calculated using electron density of three states (eqs. S3-S5):

$$
\begin{gathered}
f^{+}(r)=\rho_{N+1}(r)-\rho_{N}(r) \approx \rho^{\text {LUMO }}(r) \\
f^{-}(r)=\rho_{N}(r)-\rho_{N-1}(r) \approx \rho^{\text {HOMO }}(r) \\
\mathrm{f}^{0}(r)=\frac{f^{+}(r)+f^{-}(r)}{2}=\frac{\rho_{N+1}(r)-\rho_{N-1}(r)}{2} \approx \frac{\rho^{H O M O}(r)+\rho^{\text {LUMO }}(r)}{2}
\end{gathered}
$$

where $\mathrm{N}$ is the number of electrons in the current molecular system. The $N-1$ and $N+1$ states share the same molecular geometry as the $N$ state. For nucleophiles, $f^{-}$ is the reactivity descriptor, while for electrophiles, $f^{+}$is the descriptor. $f^{0}$ is reactivity descriptor for radical attack. Atoms with larger Fukui function tend to have higher reactivities. Isosurface maps were produced using VMD 1.9.3 program based on outputs from the Multiwfn calculations.

Molecular polarity index (MPI) was also calculated, for which is expressed as (eqs. S6):

$$
\text { MPI }=(1 / A) \iint_{S}|V(r)| d S
$$

where A and $V(r)$ refer to the area of vdW surface and value of ESP at a point $r$ in space, respectively. The integration is performed over the whole molecular vdW surface (S). MPI is a quite reliable index of measuring molecular polarity, the larger the index, the higher the polarity. ${ }^{23}$

(A)

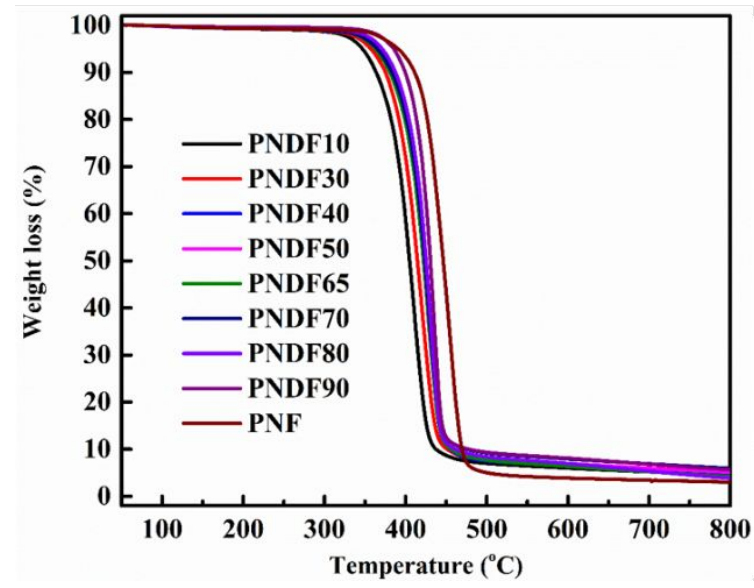

(B)

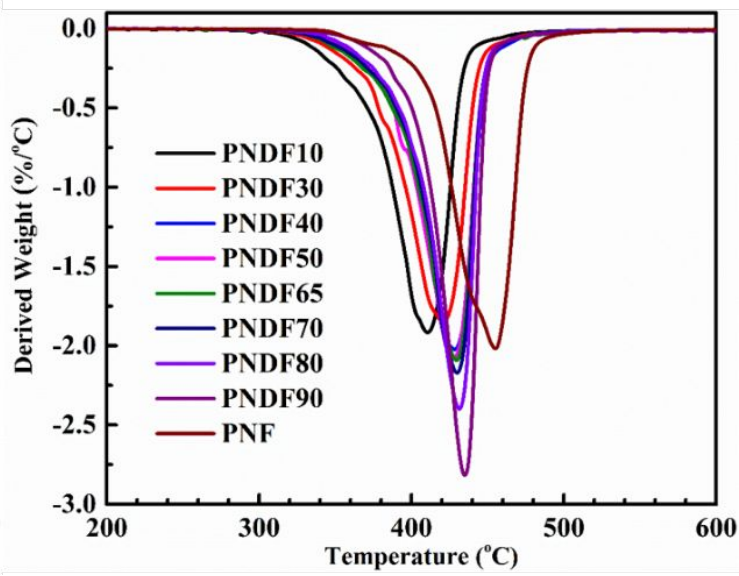

Fig. S1 (A) TGA and (B) DTG curves of PNDFs. 
Table S1. Thermal properties of PNDFs.

\begin{tabular}{|c|c|c|c|c|c|c|c|c|c|c|c|}
\hline \multirow[t]{2}{*}{ Sample } & \multicolumn{2}{|c|}{$1^{\text {st }}$ heating scan } & \multicolumn{2}{|c|}{ Cooling scan } & \multicolumn{5}{|c|}{$2^{\text {nd }}$ heating scan } & \multicolumn{2}{|c|}{ TGA } \\
\hline & $\begin{array}{c}\mathrm{T}_{\mathrm{m}} \\
\left({ }^{\circ} \mathrm{C}\right)\end{array}$ & $\begin{array}{c}\Delta \mathrm{H}_{\mathrm{m}} \\
(\mathrm{J} / \mathrm{g})\end{array}$ & $\begin{array}{c}\mathrm{T}_{\mathrm{c}} \\
\left({ }^{\circ} \mathrm{C}\right)\end{array}$ & $\begin{array}{l}\Delta \mathrm{H}_{\mathrm{c}} \\
(\mathrm{J} / \mathrm{g})\end{array}$ & $\begin{array}{c}\mathrm{T}_{\mathrm{g}} \\
\left({ }^{\circ} \mathrm{C}\right)\end{array}$ & $\begin{array}{c}\mathrm{T}_{\mathrm{cc}} \\
\left({ }^{\circ} \mathrm{C}\right)\end{array}$ & $\begin{array}{l}\Delta \mathrm{H}_{\mathrm{cc}} \\
(\mathrm{J} / \mathrm{g})\end{array}$ & $\begin{array}{c}\mathrm{T}_{\mathrm{m}} \\
\left({ }^{\circ} \mathrm{C}\right)\end{array}$ & $\begin{array}{l}\Delta \mathrm{H}_{\mathrm{m}} \\
(\mathrm{J} / \mathrm{g})\end{array}$ & $\begin{array}{l}\mathrm{T}_{5 \%} \\
(\%)\end{array}$ & $\begin{array}{c}T_{\mathrm{d}, \max } \\
(\%)\end{array}$ \\
\hline PNDF10 & 84.1 & 1.6 & I & I & 4.0 & 1 & I & I & I & 347 & 410 \\
\hline PNDF30 & 54.2 & 0.2 & l & l & 16.1 & / & / & / & / & 357 & 421 \\
\hline PNDF40 & l & / & l & / & 23.5 & / & / & / & / & 365 & 428 \\
\hline PNDF50 & / & / & / & / & 28.0 & / & / & / & / & 369 & 427 \\
\hline PNDF65 & l & / & l & / & 42.2 & l & / & / & l & 362 & 430 \\
\hline PNDF70 & I & / & / & / & 45.4 & / & / & / & / & 365 & 430 \\
\hline PNDF80 & 175.4 & 7.2 & / & / & 54.7 & 144.9 & 6.8 & 175.0 & 6.8 & 371 & 431 \\
\hline PNDF90 & 191.4 & 27.9 & / & / & 64.8 & 144.4 & 27.8 & 190.2 & 27.7 & 385 & 434 \\
\hline PNF & 202.5 & 29.5 & 1 & I & 70.7 & 146.0 & 32.7 & 200.9 & 32.5 & 391 & 455 \\
\hline
\end{tabular}

Table S2. Mechanical properties of PNDFs.

\begin{tabular}{cccc} 
Sample & $\mathbf{E}(\mathbf{M P a})$ & $\left.\boldsymbol{\sigma}_{\mathbf{b}} \mathbf{( M P a}\right)$ & $\boldsymbol{\varepsilon}_{\mathbf{b}}(\mathbf{\%})$ \\
\hline PNDF30 & $8 \pm 1$ & $2.3 \pm 0.2$ & $1400 \pm 20$ \\
PNDF40 & $58 \pm 2$ & $10.4 \pm 2.6$ & $1050 \pm 50$ \\
PNDF50 & $108 \pm 15$ & $10.7 \pm 0.1$ & $800 \pm 10$ \\
PNDF65 & $1665 \pm 23$ & $45.5 \pm 6.4$ & $340 \pm 22$ \\
PNDF70 & $1887 \pm 32$ & $58.6 \pm 4.7$ & $14 \pm 4$ \\
PNDF80 & $1943 \pm 28$ & $61.5 \pm 1.5$ & $5 \pm 1$ \\
PNDF90 & $2265 \pm 17$ & $64.8 \pm 0.4$ & $4 \pm 1$ \\
PNF & $1977 \pm 28$ & $68.1 \pm 1.5$ & $6.0 \pm 0.6$ \\
\hline
\end{tabular}

Table S3. Recoverability of PNDF40 and PNDF50

\begin{tabular}{ccccccc}
\hline Sample & $\boldsymbol{\varepsilon}(\mathbf{\%})$ & $\mathbf{R}_{\mathbf{r}}(\mathbf{1}) \%$ & $\mathbf{R}_{\mathbf{r}}(\mathbf{2}) \%$ & $\mathbf{R}_{\mathbf{r}}(\mathbf{3}) \%$ & $\mathbf{R}_{\mathbf{r}}(\mathbf{4}) \%$ & $\mathbf{R}_{\mathbf{r}} \mathbf{( 5 )} \%$ \\
\hline PNDF40 & 200 & 62.1 & 93.0 & 90.6 & 96.6 & 98.9 \\
PNDF50 & 200 & 51.7 & 86.0 & 72.0 & 92.8 & 100.0 \\
\hline
\end{tabular}

Table S4. Gas and water vapor permeability coefficients for PNDFs, other polymers for comparison.

\begin{tabular}{ccccccc}
\hline $\begin{array}{c}\text { Sample } \\
\text { [a] }\end{array}$ & $\begin{array}{c}\mathrm{CO}_{2} \\
\text { (barrer) }{ }^{[\mathrm{b}]}\end{array}$ & $\mathrm{BIFp}$ & $\begin{array}{c}\mathrm{O}_{2} \\
(\text { barrer })^{[\mathrm{c}]}\end{array}$ & $\mathrm{BIFp}$ & $\begin{array}{c}\mathrm{H}_{2} \mathrm{O} \\
\left(\mathrm{g} \cdot \mathrm{cm} / \mathrm{cm}^{2} \cdot \mathrm{s} \cdot \mathrm{Pa}\right)\end{array}$ & $\mathrm{BIFp}$ \\
\hline PBAT & 5.9 & 1 & 0.76 & 1 & $3.52 \times 10^{-13}$ & 1 \\
PNDF40 & 0.043 & 136.8 & 0.050 & 15.3 & $1.43 \times 10^{-13}$ & 2.5 \\
PNDF50 & 0.032 & 184.3 & 0.039 & 19.5 & $7.62 \times 10^{-14}$ & 4.6 \\
PNDF70 & 0.033 & 178.8 & 0.041 & 18.5 & $4.72 \times 10^{-14}$ & 7.5 \\
PNDF80 & 0.038 & 155.3 & 0.036 & 21.1 & $4.40 \times 10^{-14}$ & 8.0 \\
PNDF90 & 0.035 & 168.6 & 0.033 & 23.0 & $3.68 \times 10^{-14}$ & 9.6 \\
\hline
\end{tabular}




\begin{tabular}{ccccccc}
\hline PNF & 0.040 & 147.5 & 0.035 & 21.7 & $2.58 \times 10^{-14}$ & 13.6 \\
PLA & 1.0 & 5.9 & 0.25 & 3.0 & $1.10 \times 10^{-13}$ & 3.2 \\
PEF & 0.010 & 590 & 0.011 & 69.1 & - & - \\
PET & 0.130 & 45.4 & 0.060 & 12.7 & - & - \\
\hline
\end{tabular}

[a] The test performed at low pressure $(0.1001 \mathrm{MPa})$. ${ }^{[b]} \mathrm{CO}_{2}$ permeability coefficient, at $23{ }^{\circ} \mathrm{C}, 50 \%$ relative humidity. 1 barrer $=10^{-10} \mathrm{~cm}^{3} \cdot \mathrm{cm} / \mathrm{cm}^{2} \cdot \mathrm{s} \cdot \mathrm{cmHg}$. ${ }^{[c]} \mathrm{O}_{2}$ permeability coefficient, at $23{ }^{\circ} \mathrm{C}, 50 \%$ relative humidity. [d] Water vapor transmission rate, at $38{ }^{\circ} \mathrm{C}, 90 \%$ relative humidity.

Table S5. Molecular weight changes of PNDFs after 42 days of hydrolysis.

\begin{tabular}{lcccccc}
\hline & \multicolumn{2}{c}{$\begin{array}{c}\text { Before } \\
\text { degradation }\end{array}$} & \multicolumn{5}{c}{ After 42 days of hydrolysis } \\
\cline { 3 - 7 } Sample & \multicolumn{3}{c}{$37^{\circ} \mathrm{C}$} & \multicolumn{3}{c}{$50^{\circ} \mathrm{C}$} \\
& $\mathrm{M}_{\mathrm{w}}(\mathrm{g} / \mathrm{mol})$ & $\mathrm{DI}$ & $\mathrm{M}_{\mathrm{w}}(\mathrm{g} / \mathrm{mol})$ & $\mathrm{DI}$ & $\mathrm{M}_{\mathrm{w}}(\mathrm{g} / \mathrm{mol})$ & $\mathrm{DI}$ \\
\cline { 2 - 7 } PNDF80 & $7.68 \times 10^{4}$ & 1.9 & $7.65 \times 10^{4}$ & 1.9 & $7.58 \times 10^{4}$ & 1.8 \\
PNDF65 & $9.48 \times 10^{4}$ & 2.0 & $8.87 \times 10^{4}$ & 2.0 & $6.25 \times 10^{4}$ & 1.9 \\
PNDF40 & $9.98 \times 10^{4}$ & 1.7 & $7.32 \times 10^{4}$ & 1.8 & $4.21 \times 10^{4}$ & 1.9 \\
PNDF30 & $1.21 \times 10^{5}$ & 1.8 & $6.85 \times 10^{4}$ & 2.2 & $1.35 \times 10^{4}$ & 2.3 \\
PNDF10 & $1.33 \times 10^{5}$ & 1.9 & $3.25 \times 10^{4}$ & 2.1 & $8.34 \times 10^{3}$ & 2.2 \\
\hline
\end{tabular}

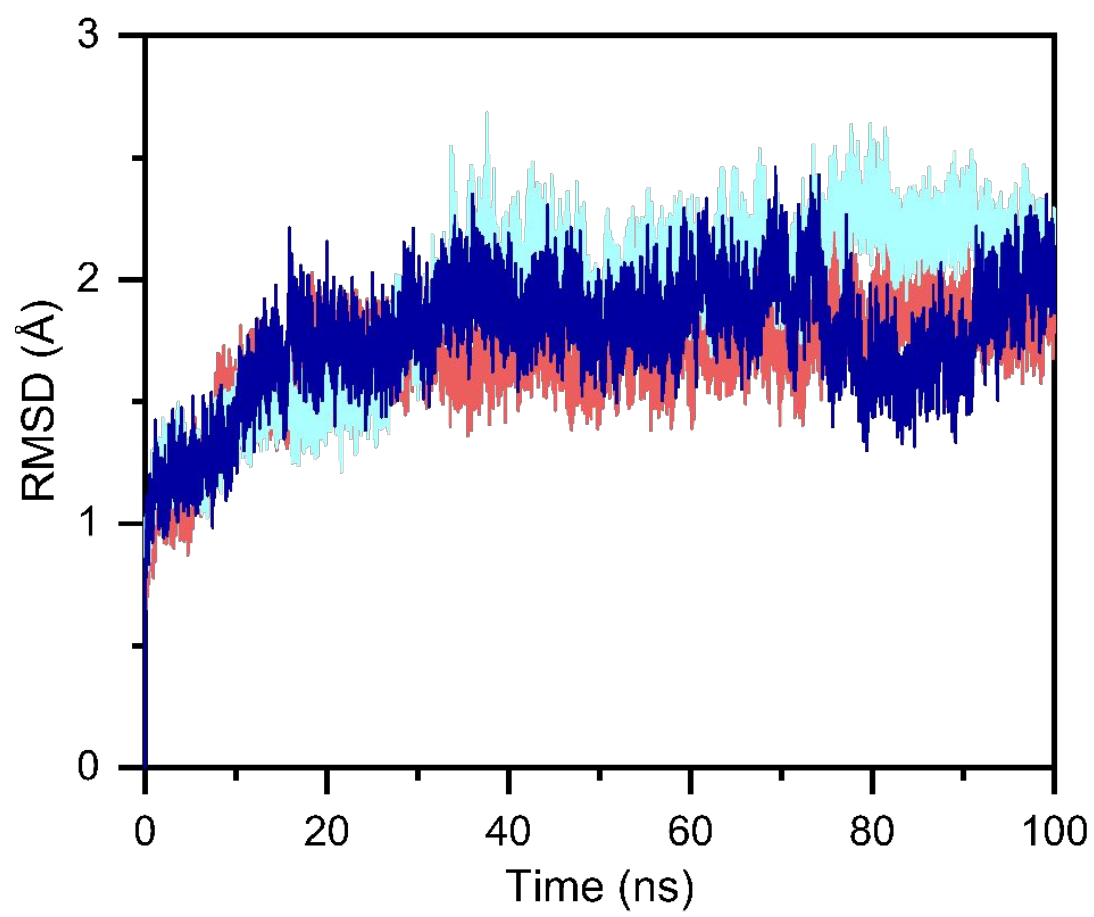

Fig. S2 Root-mean-square deviation (RMSD) of the backbone carbon atom in PNDFCALB. The three substrate-enzyme systems were each constructed and performed three times in $100 \mathrm{~ns}$ molecular dynamic (MD) simulations, respectively. Stable RMSD of 
the protein till the end of the simulation, suggested that the simulations were suitable for further rigorous analysis.
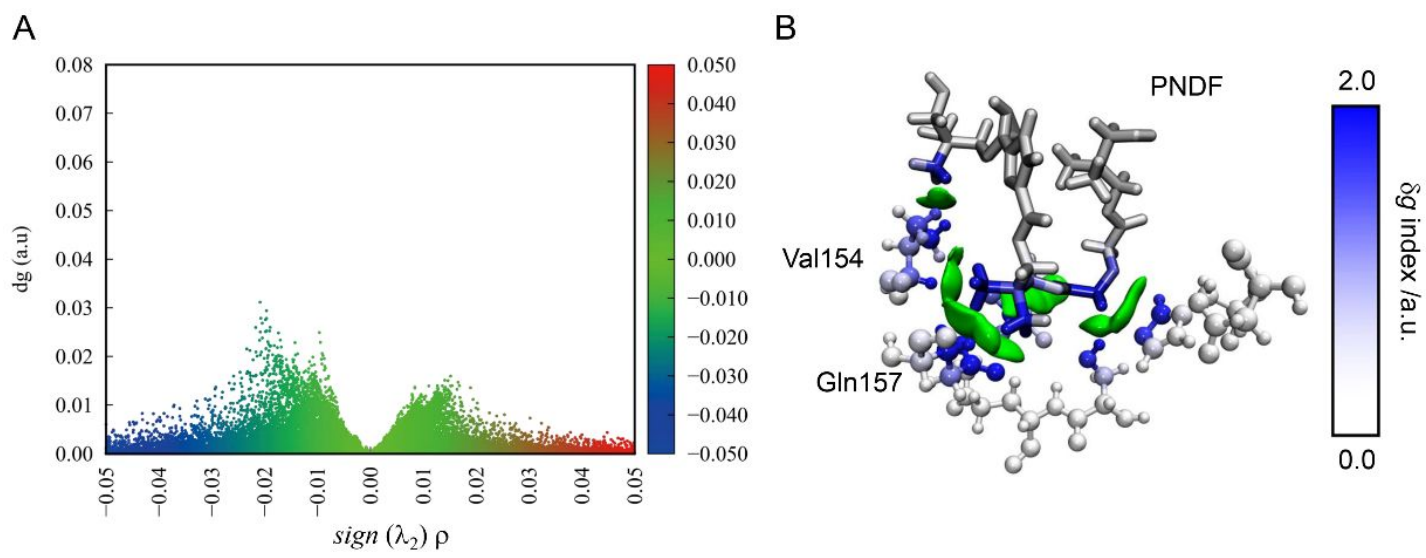

Fig. S3 Intermolecular non-covalent interaction in the PNDF-CALB. Plotting sign $\left(\lambda_{2}\right) \rho$ colored IGM scatter map (A). The atoms of CALB interface colored according to their contributions to the binding with PNDF (B). White indicates no contribution to the complexation, and blue indicates the largest relative contribution

Table S6: Comparison of the values of Hirshfeld charges and Fukui functions for Carbonyl carbon atom of ester groups in PNDF50. The atom owing larger values of $f^{+}$ became the favorable sites for nucleophilic attack.

\begin{tabular}{lllcl}
\hline Atom & $\mathbf{q ( N )}$ & $\mathbf{q}(\mathbf{N}+\mathbf{1})$ & $\mathbf{q}(\mathbf{N}-1)$ & $\mathrm{f}^{+}$ \\
\hline $\mathrm{C} 1$ & 0.2255 & 0.1542 & 0.2308 & 0.0713 \\
$\mathrm{C} 2$ & 0.2137 & 0.2145 & 0.2177 & 0.0007 \\
$\mathrm{C} 3$ & 0.1896 & 0.1388 & 0.1992 & 0.0508 \\
$\mathrm{C} 4$ & 0.1982 & 0.1626 & 0.2077 & 0.0356 \\
\hline
\end{tabular}

Table S7: Comparison of the values of Hirshfeld charges and Fukui functions for Carbonyl carbon atom of ester groups in PNDF65. The atom owing larger values of $f^{+}$ became the favorable sites for nucleophilic attack.

\begin{tabular}{lllll}
\hline Atom & $\mathbf{q}(\mathbf{N})$ & $\mathbf{q}(\mathbf{N}+\mathbf{1})$ & $\mathbf{q}(\mathbf{N}-1)$ & $\mathrm{f}^{+}$ \\
\hline $\mathrm{C} 1$ & 0.2082 & 0.2067 & 0.2141 & 0.0015 \\
$\mathrm{C} 2$ & 0.2100 & 0.2102 & 0.217 & 0.0002
\end{tabular}




\begin{tabular}{ccccc} 
C3 & 0.2199 & 0.1700 & 0.2223 & 0.0499 \\
\hline C4 & 0.2022 & 0.1627 & 0.2035 & 0.0395 \\
\hline
\end{tabular}

\section{Reference}

1. Grimme, S.; Bannwarth, C.; Shushkov, P., J. Chem. Theory Comput. 2017, 13 (5), 1989-2009.

2. Stewart, J. J. P. MOPAC2016. Steward Computational Chemistry; http://openmopac.net/ Colorado Springs: CO, USA, 2016

3. Tian Lu, molclus program, Version 1.9.4, (accessed June 18th 2020) http://www.keinsci.com/research/molclus.html

4. Marenich, A. V., Cramer, C. J. \& Truhlar, D. G, J. Phys. Chem. B, 2009, 113, 45384543.

5. Frisch, M. J.; Trucks, G. W.; Schlegel, H. B.; Scuseria, G. E.; Robb, M. A.; Cheeseman, J. R.; Scalmani, G.; Barone, V.; Petersson, G. A.; Nakatsuji, H.; Li, X.; Caricato, M.; Marenich, A. V.; Bloino, J.; Janesko, B. G.; Gomperts, R.; Mennucci, B.; Hratchian, H. P.; Ortiz, J.V.; Izmaylov, A. F.; Sonnenberg, J. L.; Williams; Ding, F.; Lipparini, F.; Egidi, F.; Goings, J.; Peng, B.; Petrone, A.; Henderson, T.; Ranasinghe, D.; Zakrzewski, V. G.; Gao, J.; Rega, N.; Zheng, G.; Liang, W.; Hada, M.; Ehara, M.; Toyota, K.; Fukuda, R.; Hasegawa, J.; Ishida, M.; Nakajima, T.; Honda, Y.; Kitao, O.; Nakai, H.; Vreven, T.; Throssell, K.; Montgomery, Jr., J. A.; Peralta, J. E.; Ogliaro, F.; Bearpark, M. J.; Heyd, J. J.; Brothers, E. N.; Kudin, K. N.; Staroverov, V. N.; Keith, T. A.; Kobayashi, R.; Normand, J.; Raghavachari, K.; Rendell, A. P.; Burant, J.C.; Iyengar, S. S.; Tomasi, J.; Cossi, M.; Millam, J. M.; Klene, M.; Adamo, C.; Cammi, R.; Ochterski, J. W.; Martin, R. L.; Morokuma, K.; Farkas, O.; Foresman, J. B.; Fox, D. J. Gaussian 16, Revision A.03; Wallingford, CT, 2016.

6. Lee, C.; Yang, W.; Parr, R. G, Phys. Rev. B: Condens. Matter Mater. Phys. 1988, 37,785 .

7. Risthaus, T.; Grimme, S, J. Chem. Theory Comput. 2013, 9, 1580-159 
8. Maeda, S.; Harabuchi, Y.; Ono, Y.; Taketsugu, T.; Morokuma, K, Int. J. Quantum Chem. 2015, 115, 258-269.

9. O. Trott, A. J. Olson, J.Comput. Chem 31 (2010) 455-46

10. W. D. Cornell, P. Cieplak, C. I. Bayly, I. R. Gould, K. M. Merz, D. M. Ferguson,

D. C. Spellmeyer, T. Fox, J. W. Caldwell and P. A. A. Kollman, J. Am. Chem. Soc., 1995, 117(19), 5179-5197

11. H. Stoll, G. Wagenblast and H. Preuss, J. Am. Chem. Soc., 1978, 100(24), 77427743.

12. Case DA, Ben-Shalom IY, Brozell SR, Cerutti DS, Cheatham TE, Cruzeiro VWD (2018) AMBER18, University of California, San Francisco.

13. J. A. Maier, C. Martinez, K. Kasavajhala, L. Wickstrom and K. E. Hauser, Simmerling, C, J. Chem. Theory Comput., 2015, 11(8), 3696-3713.

14. T. J. Dolinsky, J. E. Nielsen, J. A. McCammon and N. A. Baker, Nucleic Acids Res., 2004, 32(W), W665-W667

15. T. Darden, D. York and L. Pedersen, J. Chem. Phys., 1993, 98(12), 10089-10092.

16. J. P. Ryckaert, G. Ciccotti and H. J. C. Berendsen, J. Chem. Phys., 1977, 23(3), $327-341$.

17. Lefebvre, C., Rubez, G., Khartabil, H., Boisson, J. C., Contreras-García, J., and Hénon, E, Phys. Chem. Chem. Phys. 2017, 19, 17928-17936.

18. Lu, T.; Chen, F., J. Comput. Chem. 2012, 33 (5), 580-592.

19. Humphrey, W.; Dalke, A.; Schulten, K., J. Mol. Graphics 1996, 14 (1), 33-38.

20. Wang Y, Zhang C, Zhao YL, Zhao R, Houk KN. Biomolecules. 2020, 10(6), 815.

21. Parr, R. G.; Yang, W, J. Am. Chem. Soc. 1984, 106, 4049-4050.

22. Fukui, K.; Yonezawa, T.; Shingu, H, J. Chem. Phys. 1952, 20, 722-725

23. Liu, Z., Lu, T., \& Chen, Q. Carbon,2021,171, 514-523. 